\title{
Severe aplastic anemia during osimertinib treatment in a non-small cell lung cancer patient harboring EGFR T790M mutation
}

\author{
Seo Ree Kim ${ }^{1}$, Jihyun Han $^{2}$, Jae-Ho Yoon ${ }^{3}$, Jeong-Oh Kim ${ }^{4}$ and Jin hyoung Kang1,4* \\ ${ }^{1}$ Division of Medical Oncology, Department of Internal Medicine, College of Medicine, The Catholic University of Korea, Seoul, Korea \\ ${ }^{2}$ Department of Internal Medicine, College of Medicine, The Catholic University of Korea, Seoul, Korea \\ ${ }^{3}$ Department of hematology, Leukemia research institute, Catholic Hematology Hospital, Seoul St. Mary's Hospital, College of Medicine, The catholic University \\ of Korea, Seoul, Korea \\ ${ }^{4}$ Laboratory of Medical Oncology, Cancer Research Institute, College of Medicine, The Catholic University of Korea, Seoul, Republic of Korea
}

\begin{abstract}
We report a severe aplastic anemia occurring during the administration of osimertinib in a lung adenocarcinoma patient carrying EGFR T790M mutation.
\end{abstract}

\section{Introduction}

For lung cancer patients harboring epidermal growth factor receptor (EGFR) mutations, targeting agents to competitively inhibit EGFR tyrosine kinase are recommended as the first-line treatment. If an increase in the primary lesion is seen during the period of medication, re-biopsy should be performed to confirm the acquired resistance. and If T790M acquired resistance is accompanied, clinicians should consider starting the 3 rd tyrosine kinase inhibitor (TKI) to counteract that.

Osimertinib, a third generation targeting agent, has excellent activity and mild toxicity than the first- or second-generation EGFR targeting agents due to high selectivity, as well as good blood brain barrier permeability. Herein, we report severe aplastic anemia occurring during the administration of osimertinib in a lung adenocarcinoma patient carrying EGFR T790M mutation.

\section{Case presentation}

A 67-year-old man was diagnosed with stage IVa (pT2N1M1a) lung adenocarcinoma. The tumor was a $3.4 \mathrm{~cm}$ mass in the right upper lobe accompanied by pleural seeding nodules. Palliative chemotherapy including gemcitabine $\left(1.25 \mathrm{mg} / \mathrm{m}^{2}\right.$ on days 1 and 8 , every three weeks) and cisplatin $\left(75 \mathrm{mg} / \mathrm{m}^{2}\right.$ on day 1 , every three weeks) was immediately administered prior to checking the result of EGFR mutation test. The patient underwent six cycles of cytotoxic chemotherapy and the tumor shrinkage reached partial response. However, the response duration following the completion of chemotherapy was not much longer than our expectation. We decided to give gefitinib (250mg orally, once a day), an EGFR targeting agent, as a second-line treatment.

After 3.2 years of gefitinib treatment, two nodules were gradually increased in the right upper lobe, and a metastasis lesion was found in the S6 segment of the liver in a chest and abdomen CT scan, suggestive of disease progression. To determine the possible acquired resistance to gefitinib, re-biopsy was necessary. We performed CT- guided percutaneous core needle biopsy on Nov. 2017. A molecular study revealed that exon 19 deletion and exon 20 insertion mutation andT790M mutation coexisted. Based on this result, we began treating the patient with osimertinib (80mg orally, once a day) on Dec. 2017.

Only one month after beginning the osimertinib treatment, the primary tumor and pleural seeding nodules were markedly decreased, and a metastatic lesion around the sixth segment of the liver was also completely regressed. However, after 19 weeks of osimertinib treatment, we found that the patient's platelet count was greatly reduced to $40,000 \times 10^{6}$ cells/L (grade 3 , thrombocytopenia) in a blood test. We decided to cease osimertinib treatment and transfused platelet concentrates on Apr. 2018. The patient experienced a periodic rise and fall in platelet counts as rare as once in a blue moon, but thrombocytopenia, agranulocytosis, and anemia gradually progressed. After 21 weeks of osimertinib treatment, grade 4 thrombocytopenia and grade 3 neutropenia were detected, and platelet transfusion was immediately carried out with granulocyte colony stimulating factors (Filgrastim 150mcg subcutaneously) (Figure 1).

Despite hospitalization and active supportive care, the patient's blood count was refractory to platelet transfusion and G-CSF administration, even four weeks after the discontinuation of osimertinib. He showed that the appearance of a new-onset pancytopenia can be caused by a wide variety of etiology, leading to a diagnostic dilemma.

*Correspondence to: Jin-Hyoung Kang MD, $\mathrm{PhD}$, Department of Medical Oncology, Seoul St. Mary's Hospital, The Catholic University of Korea, 222, Banpo-daero, Seocho-gu, Seoul, 06591, Korea, Phone: +82-2-2258-6043; E-mail: oncologykang@naver.com

Key words: epidermal growth factor, receptor T790M mutation, Osimertinib, aplastic anemia, eltrombopag

Received: September 16, 2018; Accepted: September 24, 2018; Published: September 27, 2018 


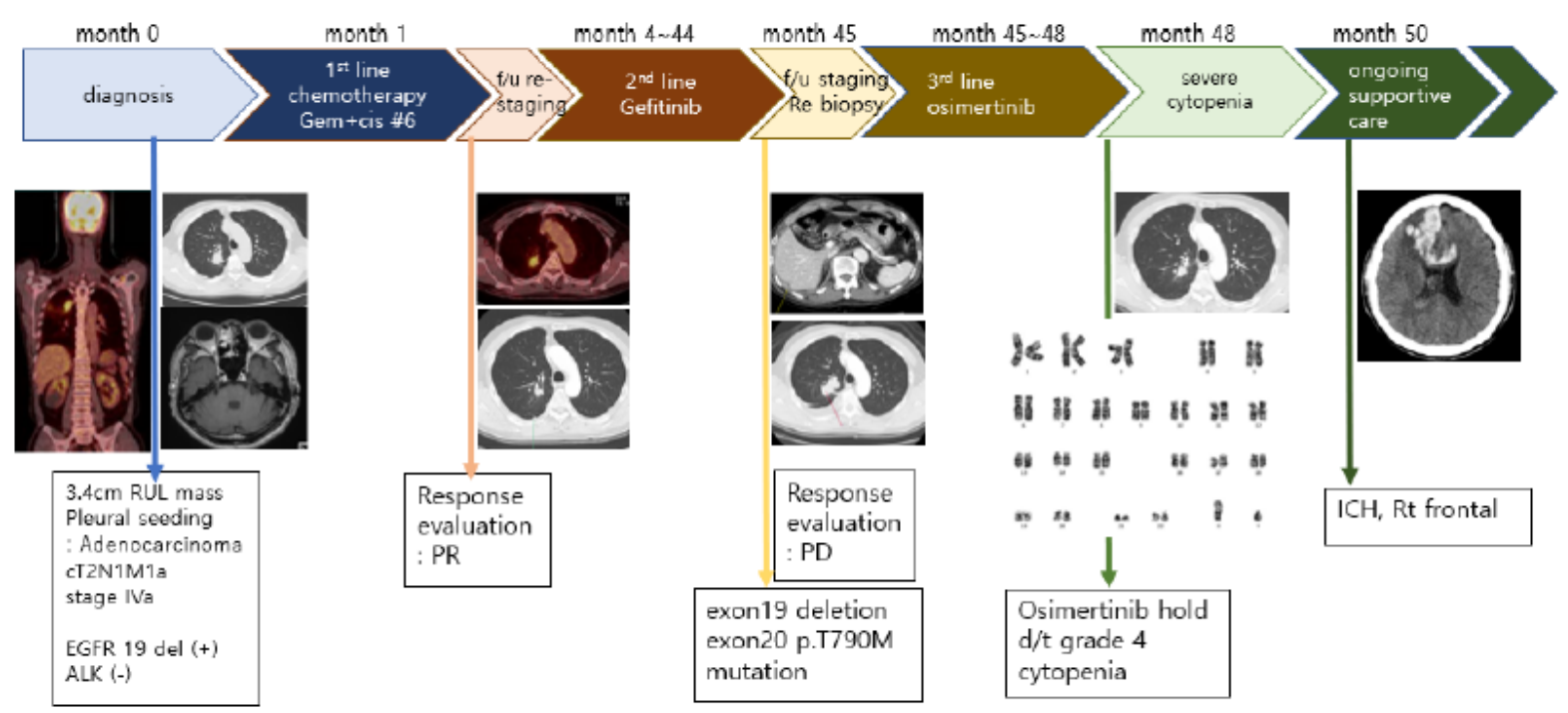

Figure 1. Filgrastim 150mcg subcutaneously

These etiologies range from drug (chemical)-induced bone marrow failure, marrow space-occupying lesions, infections, autoimmune disorder, and peripheral destruction of hematopoietic cells. In our case, the patient's blood cell count was normal for three years or more, so we could assume it was caused by an acquired factor.

To differentiate these various causes, we analyzed multiple laboratory profiles. However, there was no evidences of autoimmune diseases such as systemic lupus erythematosus and rheumatism because ANA, p-ANCA, c-ANCA, ESR, and rheumatic factor were all negative. The patient also showed negative titer of viral infections such as hepatitis profile, Brucella, EBV virus, CMV virus, Parvo virus, etc. He denied herbal medicine such as various organic acidurias or any medications other than those prescribed by the hospital. He also had no previous history of benzene, Gold, chloramphenicol, or arsenic oxidase exposure. In addition, the chromosome study was normal karyotype.

Finally, we decided to perform bone marrow aspiration and biopsy. The bone marrow cellularity was only $5 \%$ and trilineage progenitor cells were not easily visible with the fatty change entirely replaced. These findings were consistent with very severe aplastic anemia (Figure 2).

Despite the active administration of G-CSF and frequent platelet and RBC transfusion, bone marrow function was not reversed, and instead spontaneous intracranial hemorrhage occurred in the right hemisphere. The neurosurgeon recommended careful observation with active platelet transfusion instead of operation owing to the seriously reduced blood cell count. While receiving the best supportive care, intracranial hemorrhage was slightly absorbed and motor weakness of the left lower extremity gradually improved. With consideration of the severe aplastic anemia as well as the patient's age and performance status, allogenic bone marrow transplantation or strong immunosuppressant such as anti-thymoglobulin and cyclosporin was not adequate. Following an interview with the patient and his family members, we started Eltrombopag (oral thrombopoietin mimetic) on Jun 2018. Since then, his platelet count has slightly improved (Table 1).

\section{Discussion}

The development of drug resistance is a major barrier to the successful long-term treatment of EGFR TKIs for NSCLC patients with activating EGFR mutations [1,2]. Unfortunately, acquired resistance to first- and second-generation EGFR TKIs invariably arises within 9 13 months. Multiple and complex mechanisms of this acquired resistance have been identified, including secondary mutations in EGFR (e.g. EGFR-T790M), the amplification of c-MET oncogene, ERBB2 amplification, MAPK1 amplification, PIK3CA mutation, BRAF mutations, AXL activation, and small cell transformation [3-7]. The most common of the various acquired resistance mechanisms is EGFR T790M, presenting on more than $50 \%$ of repeat biopsies [8,9].

Osimertinib, a highly selective and irreversible EGFR TKI, is known to be highly active for NSCLC patients harboring either activating EGFR mutations or T790M resistance mutation. For that reason, when disease progression is generated in a lung cancer patient who has been treated with EGFR TKIs, re-biopsy should be considered for the purpose of identifying EGFR T790M mutation [9]. Phase I, II, and III clinical studies of osimertinib (AURA 1-3) have shown high objective response rates, ranging from $57-71 \%$ with favorable clinical outcomes [6-8]. Based on its safety profile collected from several clinical studies, osimertinib causes milder dermatologic and gastro-intestinal toxicities compared to historical data on and clinical experiences of other EGFR TKIs. As is commonly known, the typical adverse events of osimertinib included diarrhea (47\%), rash (40\%), nausea $(22 \%)$, and decreased appetite $(21 \%)$, and hematologic abnormality $(11 \%)$. However, the frequency of grade 3-5 serious hematologic toxicities was less than $2 \%$ $[1,9]$. Severe hematologic toxicity such as aplastic anemia is extremely rare and only one case report is present in the literature [10].

Aplastic anemia, defined as pancytopenia with a fatty or "empty" bone marrow, is characterized in context by the simplicity of its pathologic feature and the direct derivation of its clinical manifestations [11]. With regard to the pathophysiology of aplastic anemia, secondary acquired disease is more common than the primary disease. In acquired aplastic anemia, bone marrow failure results from immunologically mediated, tissue-specific organ destruction [12]. Following exposure to an irritating antigen, cells and cytokines of the immune system act destructively on stem cells in the marrow, ultimately leading to a reduction of their number so that normal levels of circulating leukocytes, erythrocytes, and platelets are not able to be maintained [11]. In the present case, osimertinib may act as an irritating antigen. 
Table 1. The hematologic parameters prior to, during, and after osimertinib treatment

\begin{tabular}{|l|c|c|c|c|c|c|c|}
\hline \multicolumn{3}{|c|}{ Osimertinib } \\
\cline { 2 - 7 }
\end{tabular}

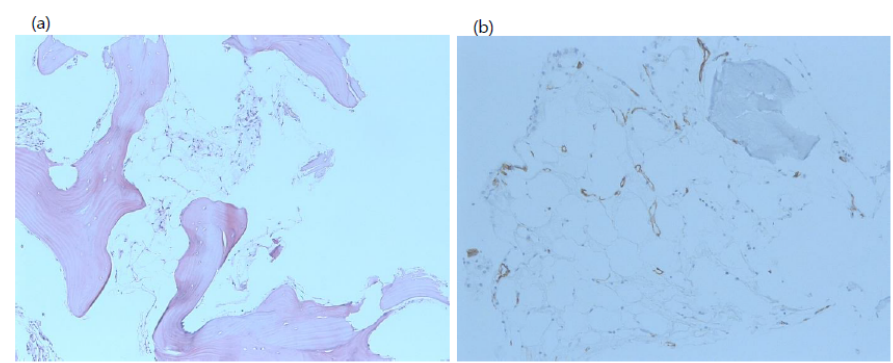

Figure 2. (a) histologic features of bone marrow after discontinuation of osimertinib treatment, Hematoxylin and eosin staining revealed marked hypocellularity (x200) (b) Immunohistochemical staining with CD34 monoclonal antibody in bone marrow biopsy specimen $(\mathrm{x} 200)$

However, it is very unlikely that it directly attacks hematopoietic progenitor cells in bone marrow, since there were not any changes in blood cells seen at the early stage of drug administration. Stromal cells provide growth factors that are essential for the viability, proliferation, and differentiation of stem cells and progenitor cells. When these cells are attacked by irritating antigens, several cytokines and lymphokines are secreted, then secondarily suppress hematopoiesis [13].

Generally, immunosuppressive therapies or allogenic bone marrow transplantation can reverse this process, resulting in improved marrow function and the partial or full resolution of pancytopenia, although without an increased number of stem cells. If the patient does not have enough performance status to overcome such an aggressive treatment or the patient fails to respond to immunosuppressive therapy, the administration of eltrombopag, a thrombopoiesis receptor agonist, could be taken into consideration in order to overcome the severe cytopenia and profound deficit in hematopoietic stem cells and progenitor cells [14]. A recent study demonstrated thrombopoietin may increase the numbers of hematopoietic stem cells and progenitor cells [15].

In this case, whether osimertinib was a direct cause or an indirect cause, it is reasonable that this drug may contribute greatly to the suppression of stromal cells in bone marrow. Taken together with a previous case report by Okamoto, our case presents severe aplastic anemia occurring during osimertinib treatment for an advanced lung cancer patient with EGFR T790M mutation. In conclusion, because osimertinib can act as a potent irritating antigen against stromal cells in bone marrow, clinicians should be careful about changes in blood cell count during the use of this agent.

\section{References}

1. Chu CY, Choi J, Eaby-Sandy B, Langer CJ, Lacouture ME (2018) Osimertinib: A Novel Dermatologic Adverse Event Profile in Patients with Lung Cancer. The oncologist 23: 891-899.

2. Jänne PA, Yang JC, Kim DW, Planchard D, Ohe Y, et al. (2015) AZD9291 in EGFR inhibitor-resistant non-small-cell lung cancer. N Engl J Med 372: 16891699. [Crossref]

3. Nguyen KS, Kobayashi S, Costa DB (2009) Acquired resistance to epidermal growth factor receptor tyrosine kinase inhibitors in non-small-cell lung cancers dependent on the epidermal growth factor receptor pathway. Clinical lung cancer 10: 281-289.

4. Ji W, Choi CM, Rho JK, Jang SJ, Park YS, et al. (2013) Mechanisms of acquired resistance to EGFR-tyrosine kinase inhibitor in Korean patients with lung cancer. BMC cancer 13: 606 .

5. Yu HA, Arcila ME, Rekhtman N, Sima CS, Zakowski MF, et al. (2013) Analysis of tumor specimens at the time of acquired resistance to EGFR-TKI therapy in 155 patients with EGFR-mutant lung cancers. Clin Cancer Res 19: 2240-2247.

6. Ercan D, Choi HG, Yun CH, Capelletti M, Xie T, et al. (2015) EGFR Mutations and Resistance to Irreversible Pyrimidine-Based EGFR Inhibitors. Clin Cancer Res 21: 3913-3923.

7. Stewart EL, Tan SZ, Liu G, Tsao MS (2015) Known and putative mechanisms of resistance to EGFR targeted therapies in NSCLC patients with EGFR mutations-a review. Transl Lung Cancer Res 4: 67-81.

8. Jänne PA, Yang JC, Kim DW, Planchard D, Ohe Y, et al. (2015) AZD9291 in EGFR inhibitor-resistant non-small-cell lung cancer. NEngl J Med 372: 1689-1699. [Crossref]

9. Gao X, Le X, Costa DB (2016) The safety and efficacy of osimertinib for the treatment of EGFR T790M mutation positive non-small-cell lung cancer. Expert Rev Anticancer Ther 16: 383-390.

10. Ogata H, Yamamoto Y, Harada T, Nakanishi Y, Okamoto I, et al. (2017) Severe Aplastic Anemia during Osimertinib Therapy in a Patient with EGFR Tyrosine Kinase Inhibitor-Resistant Non-Small Cell Lung Cancer. J Thorac Oncol 12: e46-e7.

11. Young NS, Maciejewski J (1997) The pathophysiology of acquired aplastic anemia. $N$ Engl J Med 336: 1365-1372. [Crossref]

12. Kaufman DW, Kelly JP, Jurgelon JM, Anderson T, Issaragrisil S, et al. (1996) Drugs in the aetiology of agranulocytosis and aplastic anaemia. Eur J Haematol Suppl 60: 23-30.

13. Marsh JC (2000) Hematopoietic growth factors in the pathogenesis and for the treatment of aplastic anemia. Semin Hematol 37: 81-90. [Crossref]

14. Townsley DM, Scheinberg P, Winkler T, Desmond R, Dumitriu B, et al. (2017) Eltrombopag Added to Standard Immunosuppression for Aplastic Anemia. $N$ Engl $J$ Med 376: 1540-1550. [Crossref]

15. Lum SH, Grainger JD (2016) Eltrombopag for the treatment of aplastic anemia: current perspectives. Drug Des Devel Ther 10: 2833-2843. [Crossref]

Copyright: (C2018 Kim SR. This is an open-access article distributed under the terms of the Creative Commons Attribution License, which permits unrestricted use, distribution, and reproduction in any medium, provided the original author and source are credited. 